\title{
RELACIONES DE LA RESILIENCIA CON EL AUTOCONCEPTO Y EL APOYO SOCIAL PERCIBIDO EN UNA MUESTRA DE ADOLESCENTES
}

\section{RELATIONS OF RESILIENCE TO SELF-CONCEPT AND PERCEIVED SOCIAL SUPPORT IN A SAMPLE OF ADOLESCENTS}

\author{
ARANTZAZU RODRÍGUEZ-FERNÁNDEZ ${ }^{1}$, ESTIBALIZ RAMOS-DÍAZ ${ }^{1}$, \\ IKER $\operatorname{ROS}^{1}{ }^{1}$ ARANTZA FERNÁNDEZ-ZABALA ${ }^{1}$
}

Cómo referenciar este artículo/How to reference this article:

Rodríguez-Fernández, A., Ramos-Díaz, E., Ros, I. y Fernández-Zabala, A. (2015). Relaciones de la resiliencia con el autoconcepto y el apoyo social percibido en una muestra de adolescentes [Relations of Resilience to self-concept and perceived social support in a simple of adolescents]. Acción Psicológica, 12(2), 1-14. doi: http://dx.doi.org/10.5944/ap.12.2.14903

\section{Resumen}

El estudio científico de la resiliencia constituye en la actualidad una temática de gran interés en el ámbito de la psicología al aportar una renovada y positiva visión del desarrollo humano. Determinar los factores individuales y contextuales que influyen sobre la resiliencia durante la etapa evolutiva de la adolescencia resulta esencial para promover el ajuste de los adolescentes. El objetivo de este trabajo fue, en primer lugar, explorar las relaciones de la resiliencia con el autoconcepto y el apoyo social percibido; y, en segundo lugar, identificar variables predictoras de la resiliencia. La muestra estaba configurada por 1.250 adolescentes $(M=13.72, D T=$ 1.09). Se administraron los siguientes instrumentos de evaluación: la Escala de Resiliencia de Connor y Davidson (CD-RISC), el Cuestionario Autoconcepto Dimensional (AUDIM), el Cuestionario de Apoyo Social Percibido de Familia y Amigos (AFA) y la Escala de

Agradecimientos. Los autores del presente estudio forman parte del Grupo Consolidado de Investigación IT701-13 del Sistema Universitario Vasco. El estudio se llevó a cabo como parte del proyecto de investigación EHUA 13/26 en la Universidad del País Vasco.

Correspondencia: Estibaliz Ramos-Díaz. Departamento de Psicología Evolutiva y de la Educación, Facultad de Psicología, Universidad del País Vasco (UPV/EHU), España

Email: estibaliz.ramos@ehu.eus

${ }^{1}$ Universidad del País Vasco (UPV/EHU), España

Recibido: 23 de junio de 2015

Aceptado: 12 de octubre de 2015 
Apoyo del Profesorado (HBSC-2006). Se hallaron correlaciones positivas de la resiliencia con el autoconcepto y el apoyo social percibido, así como una variación significativa en las puntuaciones de ambas variables en función de la resiliencia. El autoconcepto y el apoyo social percibido fueron identificados como predictores de la resiliencia.

Palabras clave: resiliencia; autoconcepto; apoyo social percibido; adolescencia; psicología positiva.

Abstract
The scientific study of resilience is a subject of great
interest in the field of psychology to provide a new
and positive view of human development. To
promote adolescent adjustment, is necessary to
determine individual and contextual factors that
influence resilience during the developmental stage
of adolescence. The objective of this study was to
analyze the relations between resilience and
variables such as self-concept and social support;
and to identify variables that predict resilience. The
sample is made up of 1250 adolescents ( $M=13.72$,
$S D=1.09$ ). Three assessment instruments were
administered: The Connor-Davidson Resilience
Scale (CD-RISC), the Dimensional Self-Concept
Questionnaire (AUDIM), the Perceived Social
Support from Family and Friends Questionnaire
(AFA) and the Teacher Social Suppport Scale
(HBSC-2006). Positive correlations between self-
concept and social support with resilience were
founded. Test scores of self-concept and social
support varied depending of the level of resilience.
Self-concept and social support were predictor
variables of resilience.

Keywords: resilience; self-concept; perceived social support; adolescence; positive psychology.

\section{Introducción}

Hasta finales del siglo pasado, los campos de la psicología y la educación han manifestado más interés por identificar y remediar los desajustes de la adolescencia, que por definir las competencias sociopersonales que configuran las trayectorias evolutivas adaptativas durante el periodo de transición de la infancia a la edad adulta. Sin embargo, con la aparición de la ciencia de la psicología positiva (Seligman y Csikszentmihalyi, 2000; Sheldon y King, 2001), se ha producido un movimiento desde el enfoque basado en el déficit hasta un nuevo paradigma centrado en las capacidades y atributos positivos de los adolescentes (Lerner, Phelps, Forman y Bowers, 2009; Moore y Lippman, 2005; Oliva et al., 2010). Este nuevo modelo, denominado desarrollo positivo adolescente (positive youth development), trata de determinar los aspectos individuales y contextuales del desarrollo necesarios para una adolescencia saludable (Lerner, Bowers, Geldhof, Gestsdóttir y DeSouza, 2012; Masten, 2014).

Muchos son los elementos implicados en el desarrollo equilibrado o ajustado del ser humano, pero en la adolescencia, tal y como indican Masten y Tellegen (2012), puede escogerse como indicador significativo de la adaptación exitosa la resiliencia, concepto que ha ido ganando un considerable protagonismo en el ámbito de la psicología positiva (Luthar, Lyman y Crossman, 2014). Aunque los trabajos de investigación muestran una falta de consenso en su definición (Fletcher y Sarkar, 2013), existe acuerdo en definir la resiliencia como el afrontamiento adecuado de las tareas evolutivas propias de una determinada etapa de desarrollo a pesar de la adversidad (Becoña, 2006; Luthar, Cicchetti y Becker, 2000; Masten, 2014). Este estudio se centra en el dominio psicológico del constructo al considerar la resiliencia como un conjunto de cualidades personales positivas que mejoran la adaptación individual (Connor y Davidson, 2003). La teoría de la resiliencia relacionada con la reducción de riesgos se centra más en la eliminación de los factores implicados en los resultados desadaptativos de los adolescentes (Fergus y Zimmerman, 2005), mientras que el enfoque centrado en la resiliencia aquí utilizado hace hincapié en la construcción de habilidades y capacidades individuales que facilitan la adaptación exitosa de 
los adolescentes a pesar de circunstancias estresantes o adversas (Brooks y Brooks, 2014; Olsson, Bond, Burns, Vella-Brodrikck y Sawyer, 2003). Concretamente en la adolescencia tienen lugar los procesos de construcción de la identidad, el desarrollo de nuevas formas de pensamiento y el fortalecimiento de la red social, por lo que se trata de una etapa de desarrollo clave para la consolidación de valores y fortalezas personales (Giménez, Vázquez y Hervás, 2010). Como en cualquier momento de transición del ciclo vital, los adolescentes deberán desplegar una serie de conductas resilientes para hacer frente a diversas tareas evolutivas, desafíos o situaciones difíciles. Sin embargo, no todos los jóvenes se verán afectados de igual manera ante las circunstancias estresantes a las que están expuestos en su vida diaria, pudiéndose vincular determinados atributos individuales a mecanismos de resiliencia (Oliva, Jiménez, Parra y Sánchez Queija, 2008).

El afrontamiento adaptativo de las experiencias negativas en la adolescencia se han relacionado con diferentes factores de carácter contextual e individual (Liu, Wang y Lü, 2013; Masten, 2006; Wright, Masten y Narayan, 2013). Dentro del amplio conjunto de características psicológicas asociadas con el comportamiento adaptativo ante la adversidad, la percepción positiva de uno mismo figura como un elemento común que se repite de manera consistente en varios estudios pioneros sobre resiliencia (ver Dumont y Provost, 1999; Garmezy, Masten y Tellegen, 1984; Kidd y Shahar, 2008; Werner y Smith, 1992). El autoconcepto se trata de uno de los constructos que mayor interés ha suscitado en el campo de la psicología y ha sido ampliamente estudiado, considerándose determinante para el ajuste tanto psicológico como escolar en la adolescencia (Fuentes, García, Gracia y Lila, 2011; Garaigordobil, Pérez y Mozaz, 2008; Inglés, Martínez-Monteagudo, GarcíaFernández, Valle y Castejón, 2014). Se ha definido como un conjunto de percepciones que una persona mantiene sobre sí misma a partir de la valoración personal y de la evaluación de los otros significativos (Shalvenson, Hubner y Stanton, 1976). Tener una autopercepción negativa puede suponer un riesgo para el desarrollo adolescente. Por el contrario, tener una alta autoestima o autopercepción evaluativa positiva puede ser una cualidad protectora de resultados negativos asociados con la exposición al riesgo (Fergus y Zimmerman, 2005). En resumen, se entiende el autoconcepto como un indicador del bienestar personal que influye positivamente en las conductas ajustadas y adaptativas en la adolescencia.

Otro factor que desempeña un rol fundamental en el periodo adolescente es el apoyo social percibido, entendiendo por tal la percepción subjetiva respecto a la adecuación del soporte proporcionado por la red social (familia, amistades y otras personas del entorno). Se trata de un fenómeno complejo y dinámico que comprende un conjunto de factores en interacción y que cambia a lo largo del ciclo vital, destacando la adolescencia como un periodo que abarca diferentes etapas a través de las cuales la red social evoluciona en gran medida (Cohen, 2004). Numerosos estudios previos señalan que las personas comparten la necesidad de una respuesta positiva de aceptación por parte de personas significativas tanto a nivel interpersonal como familiar (Rohner y Carrasco, 2014). Igualmente, está ampliamente documentado mediante trabajos de investigación el papel protector del apoyo social frente a las amenazas graves al desarrollo y en favor de la adaptación de los adolescentes (Masten 2006). Se ha demostrado que el apoyo social se encuentra significativamente conectado con el desarrollo positivo en la adolescencia (Chu, Saucier y Hafner, 2010; Musitu y Cava, 2003; Rodríguez-Fernández, Droguett y Revuelta, 2012; Tian, Liu, Huang y Huebner, 2013).

En diversos estudios pertenecientes a la línea de investigación sobre la relación entre resiliencia y autoconcepto-autoestima, la visión positiva de uno mismo es señalada como factor predictor del comportamiento resiliente en niños y jóvenes (Masten, 2006; Wright, et al., 2013). Existen igualmente trabajos realizados con muestras de adolescentes y jóvenes (Benetti y Kambouropoulos, 2006; Cardozo y Alderete, 2009; Dumont y Provost, 1999; Karatas y Savi Cakar, 2011) o con menores infractores (Kidd y Shahar, 2008), que sugieren la existencia de conexión directa entre ambas variables. Puede presuponerse, por tanto, que entre los aspectos individuales que distinguen a la persona que afronta eficazmente la adversidad, la autopercepción positiva ocupa un lugar preferente. 
Se sabe menos, en cambio, sobre el rol que juega en la conducta resiliente el apoyo social. Recientemente se han aportado trabajos empíricos que corroboran el vínculo positivo entre el apoyo social y la resiliencia en la adolescencia (Bozack, 2014; Everall, Altrows y Paulson, 2006; Stumblingbear-Riddle y Romans, 2012; Sun, Guan, Qin, Zhang y Fan, 2013). Por último, Wright et al. (2013) distinguen dos tipos de factores promotores de la resiliencia: el apoyo de la familia y el contacto prosocial con iguales y adultos mentores. Por consiguiente, el apoyo social parece desempeñar un rol de protección en la determinación de las respuestas individuales a los estresores psicosociales.

Queda claro, en definitiva, que sobre la conexión que la resiliencia mantiene con el autoconcepto y el apoyo social percibido durante el período concreto de la adolescencia, últimamente se está obteniendo información más específica. Sin embargo, el análisis simultáneo de las características individuales y los factores ecológicos en la explicación de la resiliencia de los adolescentes es todavía limitado.

En función de esta contextualización teórica, el estudio llevado a cabo plantea como objetivo estudiar las relaciones de la resiliencia con el autoconcepto y el apoyo social. Con esta finalidad, y tomando como referencia la investigación previa, se proponen 3 hipótesis:

a) Existe una conexión directa entre resiliencia y autoconcepto, de manera que los adolescentes con altas puntuaciones en resiliencia presentarán un alto autoconcepto.

b) La resiliencia y el apoyo social correlacionan significativamente, de modo que los adolescentes con altas puntuaciones en resiliencia percibirán más apoyo por parte de su entorno social.

c) Alto nivel de autoconcepto, de apoyo familiar y de apoyo de las amistades serán variables predictoras de la resiliencia.

\section{Método}

\section{Participantes}

Participan en esta investigación un total de 1.250 adolescentes escolarizados en centros educativos de la Comunidad Autónoma del País Vasco. El rango de edad fue de 12 a 15 años $(M=13.72, D T=1.09)$, y del conjunto de la muestra 612 (49\%) son chicos y 638 (51\%) chicas.

Para la selección de la muestra se aplicó una técnica de muestreo aleatorio de adolescentes escolarizados en centros educativos de la Comunidad Autónoma del País Vasco (CAPV). Se empleó el criterio de centros públicosprivados y nivel socio-económico-cultural de las familias, de tal modo que pudieran representarse los diferentes tipos de centros de la CAPV. De este modo, se seleccionaron nueve centros de titularidad privadaconcertada y cinco de titularidad pública de nivel socioeconómico-cultural diverso. La chi cuadrado de Pearson indicó que no había diferencias significativas en la segmentación del número de participantes de cada sexo en los diferentes grupos de edad $\left(\chi^{2}=4.66, p>.05\right)$, por lo que la muestra resultó ser equilibrada. Una distribución de la muestra por sexo y edad queda reflejada en la Tabla 1 .

Tabla 1

Distribución de la Muestra por Sexo y Edad

\begin{tabular}{|c|c|c|c|c|c|}
\hline \multirow[t]{2}{*}{ Sexo } & \multicolumn{4}{|c|}{ Edad } & \multirow[t]{2}{*}{ Total } \\
\hline & 12 años & 13 años & 14 años & 15 años & \\
\hline Chicos & $\begin{array}{c}114 \\
(9.1 \%)\end{array}$ & $\begin{array}{c}118 \\
(9.4 \%)\end{array}$ & $\begin{array}{c}182 \\
(14.6 \%)\end{array}$ & $\begin{array}{c}198 \\
(15.8 \%)\end{array}$ & $\begin{array}{c}612 \\
(49.0 \%)\end{array}$ \\
\hline Chicas & $\begin{array}{c}118 \\
(9.4 \%)\end{array}$ & $\begin{array}{c}154 \\
(12.3 \%)\end{array}$ & $\begin{array}{c}171 \\
(13.7 \%)\end{array}$ & $\begin{array}{c}195 \\
(15.6 \%)\end{array}$ & $\begin{array}{c}638 \\
(51.0 \%)\end{array}$ \\
\hline Total & $\begin{array}{c}232 \\
(18.6 \%)\end{array}$ & $\begin{array}{c}272 \\
(21.8 \%)\end{array}$ & $\begin{array}{c}353 \\
(28.2 \%) \\
\end{array}$ & $\begin{array}{c}393 \\
(31.4 \%) \\
\end{array}$ & $\begin{array}{c}1250 \\
(100 \%)\end{array}$ \\
\hline
\end{tabular}

Nota. $\chi^{2}=4.66, p>.05$. 


\section{Instrumentos de evaluación}

Connor-Davidson Resilience Scale (CD-RISC), de Connor y Davidson (2003). Es un cuestionario autoaplicado de 25 ítems (ejemplos: Soy capaz de adaptarme a los cambios; Puedo alcanzar mis metas) y se responde en una escala de frecuencia tipo Likert que oscila desde $0=$ nada de acuerdo a $4=$ totalmente de acuerdo. La puntuación máxima es de 100, reflejando las puntuaciones más altas mayor resiliencia. El análisis factorial desarrollado por los autores sugiere una estructura pentadimensional, aunque, al igual que otros autores (Campbell-Sill, Cohan, y Stein, 2006), en los análisis se utilizó la puntuación total de los ítems. Las características psicométricas de CD-RISC, en un estudio preliminar en población general y muestra clínica, demostraron tener una adecuada consistencia interna, fiabilidad test-retest, validez convergente y divergente (Connor y Davidson, 2003), y buena validez de constructo en población adolescente (Jorgensen y Seedat, 2008; Yu, Lau, Mak, Zhang y Lui, 2011). Para precisar la versión final de la escala se tuvo en cuenta la versión traducida al castellano proporcionada por los autores originales (Bobes, Bascaran, García-Portilla, Bousoño, Sáiz y Wallance, 2001). La consistencia interna (alpha de Cronbach) obtenida con la muestra del presente estudio y para el conjunto de los ítems fue elevada $(\alpha=$ .86).

Cuestionario de Apoyo Social percibido de Familia y Amigos (AFA) de Landero y González (2008) está compuesto por 15 ítems (ejemplos: Cuentas con alguien de tu familia que te ayude a resolver algún problema; Confías en algún amigo/a para hablar de las cosas que te preocupan) destinados a medir dos dimensiones, el apoyo de la familia (8 ítems) y el apoyo de las amistades (7 ítems). Además, ofrece también una puntuación global de apoyo social percibido mediante la suma simple de todos los ítems. El formato de respuesta corresponde a una escala tipo Likert de 5 alternativas de respuesta (donde $1=$ nunca y $5=$ siempre). Los propios autores informan de análisis posteriores que confirman la validez del cuestionario y su estructura bifactorial (González y Landero, 2014), al igual que otros estudios con población adolescente española (Fernández-Zabala, Goñi, Camino, y Zulaika, en prensa; Rodríguez-
Fernández, Ramos-Díaz, Madariaga, Arribillaga y Galende, en prensa). Las propiedades psicométricas del instrumento aportadas por la muestra del presente estudio revelan índices de consistencia interna adecuados para la dimensión apoyo de la familia $(\alpha=.82)$ y apoyo de las amistades $(\alpha=.82)$, cuyos porcentajes de varianza explicada son $47.72 \%$ y $53.04 \%$ respectivamente.

Cuestionario HBSC-2006 de Moreno et al. (2008). Este cuestionario se utiliza en la edición 2006 del estudio Health Behaviour in School Aged Children (HBSC) patrocinado por la Organización Mundial de la Salud. El instrumento está compuesto por diez factores que miden las conductas relacionadas con la salud y el desarrollo de los adolescentes escolarizados. Por la mayor relevancia que posee para este trabajo, se selecciona únicamente la subescala de apoyo del profesorado perteneciente al factor ambiente psicosocial en la escuela. Los adolescentes deben contestar a las preguntas mostrando su grado de acuerdo o desacuerdo con los ocho ítems (ejemplos: En mi colegio o instituto, cuando necesito ayuda extra, mis profesores me la dan; En mi colegio o instituto, mis profesores están interesados en mí como persona) organizados en una escala tipo Likert 5 de grados, donde $1=$ totalmente de acuerdo y $5=$ totalmente en desacuerdo. El porcentaje de varianza explicada obtenido para la escala de apoyo del profesorado en la muestra de este estudio es de $47.95 \%$ y el índice de consistencia interna es de $\alpha=.84$.

Cuestionario Autoconcepto Dimensional (AUDIM) de Fernández-Zabala, Goñi, Rodríguez-Fernández y Goñi (2015). Posee un formato de respuesta en escala Likert de cinco grados que oscila desde $1=$ falso a $5=$ verdadero y consta de 33 ítems. Consta de 11 escalas que evalúan dimensiones del autoconcepto, además de una escala general para la medida del autoconcepto, que se define como la autopercepción global de la persona. En los análisis se utilizó únicamente la dimensión general, compuesta por cinco ítems (ejemplos: Me siento a disgusto conmigo mismo/a; Me siento una persona afortunada). Los autores refrendan el cuestionario AUDIM con un valor de .97 tanto para la fiabilidad omega de McDonald como para la fiabilidad compuesta, con una varianza media extractada $=.54$ y un porcentaje de varianza total explicada del $75.51 \%$. En el presente 
estudio, la consistencia alfa obtenida para el cuestionario AUDIM se sitúa en el valor $\alpha=.82$..

\section{Procedimiento}

Varios centros fueron seleccionados aleatoriamente a partir del listado de centros educativos de la CAPV. Inicialmente se contactó con los centros seleccionados con la finalidad de presentar la investigación y proponer la participación voluntaria en la misma. Dos centros desestimaron su colaboración en la investigación, por lo que se procedió a seleccionar los nuevos centros con el mismo método. Una vez confirmada su participación, se entregaron los consentimientos informados para los padres y los adolescentes.

Posteriormente, dos investigadoras acudieron a los centros y en una sesión de 30 minutos de duración administraron a los participantes la batería de instrumentos de evaluación para medir las variables objeto de estudio. Con el propósito de mitigar respuestas en dirección de las hipótesis de la investigación, se empleó el criterio de ciego único, evitando que los estudiantes conocieran la finalidad del estudio. La confidencialidad y voluntariedad fueron asimismo garantizadas para reducir los efectos de la deseabilidad social. Ningún estudiante rechazó participar en la investigación.

\section{Análisis de datos}

Con la finalidad de analizar las relaciones que existen entre la resiliencia, el autoconcepto y el apoyo social percibido se calcularon los coeficientes de correlación de Pearson con las puntuaciones obtenidas en el CD-RISC, AFA, HBSC-2006 y AUDIM. En segundo lugar, con el objetivo de identificar variables predictoras de la resiliencia se realizó un análisis de regresión lineal múltiple, método de pasos sucesivos. Los análisis estadísticos señalados fueron realizados con el paquete estadístico SPSS 22.0 para Windows. Para el tratamiento de los valores perdidos se extrajo, a partir del algoritmo de maximización de expectativa y de la Cadena Markov Monte Carlo (MCMC), una puntuación aproximada al ítem perdido basada en el patrón de respuestas.

\section{Resultados}

\section{Resiliencia en la adolescencia: relaciones con auto- concepto y apoyo social}

Los coeficientes de correlación de Pearson de la resiliencia con el autoconcepto y las escalas de apoyo social (apoyo familiar, apoyo de las amistades y apoyo del profesorado) calculados para el conjunto de la muestra y de forma diferenciada para ambos sexos y grupos de edad (12-13 años y 14-15 años), así como las correlaciones parciales controlando el efecto del sexo y la edad, se presentan en la Tabla 2.

En primer lugar, los coeficientes obtenidos evidencian que la resiliencia mantiene relaciones estadísticamente significativas $(p<.001)$ de signo positivo con el autoconcepto y las tres dimensiones de apoyo social (apoyo familiar, apoyo de las amistades y apoyo del profesorado) tanto en la muestra total como en las

Tabla 2

Correlaciones de Pearson de Resiliencia con Apoyo de la Familia, Apoyo de las Amistades y Apoyo del Profesorado y Correlaciones Parciales controlando Sexo y Edad

\begin{tabular}{|c|c|c|c|c|c|c|}
\hline & $\begin{array}{c}\text { Muestra total } \\
(N=1250)\end{array}$ & $\begin{array}{c}\text { Controlando } \\
\text { efecto sexo y } \\
\text { edad } \\
(\mathrm{N}=1250)\end{array}$ & $\begin{array}{c}\text { Chicos } \\
(N=612)\end{array}$ & $\begin{array}{l}\text { Chicas } \\
(N=638)\end{array}$ & $\begin{array}{l}12-13 \text { años } \\
(N=503)\end{array}$ & $\begin{array}{l}14-15 \text { años } \\
(N=745)\end{array}$ \\
\hline \multicolumn{7}{|c|}{ Resiliencia } \\
\hline Autoconcepto & $.451^{\star *}$ & $.436^{\star *}$ & $.412^{\star *}$ & $.466^{\star *}$ & $.329^{* *}$ & $.496^{* *}$ \\
\hline Apoyo familiar & $.278^{* *}$ & $.281^{* *}$ & $.266^{\star *}$ & $.314^{\star *}$ & $.263^{\star *}$ & $.273^{\star *}$ \\
\hline Apoyo amistades & $.207^{* *}$ & $.277^{* *}$ & $.282^{* *}$ & $.264^{* *}$ & $.183^{* *}$ & $.229^{\star *}$ \\
\hline Apoyo profesorado & $.212^{* *}$ & $200 * *$ & $.160^{\star *}$ & $280^{* *}$ & $.194^{\star *}$ & $.194^{* *}$ \\
\hline
\end{tabular}


Tabla 3

Medias y Desviaciones Típicas del Autoconcepto, Apoyo Familiar, Apoyo de las Amistades y Apoyo del Profesorado en los tres Perfiles de Resiliencia. Resultados del Análisis de Varianza en función del Perfil, Tamaño del Efecto y Pruebas Post-Hoc

\begin{tabular}{|c|c|c|c|c|c|c|c|c|}
\hline & \multicolumn{2}{|c|}{$\begin{array}{l}\text { Resiliencia baja } \\
\quad(n=204)\end{array}$} & \multicolumn{2}{|c|}{$\begin{array}{l}\text { Resiliencia media } \\
\qquad(n=857)\end{array}$} & \multicolumn{2}{|c|}{$\begin{array}{l}\text { Resiliencia alta } \\
\quad(n=189)\end{array}$} & \multirow[t]{2}{*}{$\begin{array}{c}F_{(4,1250)} \\
\text { perfil }\end{array}$} & \multirow[t]{2}{*}{ Eta } \\
\hline & $M_{\text {post-hoc }}$ & $(D T)$ & $M_{\text {post-hoc }}$ & $(D T)$ & $M_{\text {post-hoc }}$ & $(D T)$ & & \\
\hline Autoconcepto & $17.62^{\mathrm{a}}$ & $(3.94)$ & $20.45^{b}$ & $(2.99)$ & $22.19^{\mathrm{C}}$ & $(2.57)$ & $111.05^{*}$ & .153 \\
\hline Apoyo familiar & $30.46^{\mathrm{a}}$ & (6.21) & $33.28^{\mathrm{b}}$ & $(4.80)$ & $34.75^{\mathrm{c}}$ & $(4.36)$ & $40.56^{*}$ & .073 \\
\hline Apoyo amistades & $26.75^{\mathrm{a}}$ & (5.43) & $28.39^{\mathrm{b}}$ & $(4.24)$ & $29.69^{C}$ & (4.47) & $22.09^{*}$ & .053 \\
\hline Apoyo profesorado & $23.89^{\mathrm{a}}$ & (5.99) & $26.65^{\mathrm{b}}$ & $(5.64)$ & $27.97^{\mathrm{C}}$ & (5.94) & $27.37^{*}$ & .070 \\
\hline
\end{tabular}

Nota. Resiliencia baja (percentil < 20), resiliencia media (percentil 20-80), resiliencia alta (percentil > 80). Eta: tamaño del efecto. La regla de Cohen (1988) señala que: .01 - .06 (efecto pequeño), .06 - .14 (efecto medio), > .14 (efecto grande). Para cada una de las variables, los tres perfiles de resiliencia con distinto superíndice indican diferencias estadísticamente significativamente en las comparaciones Post-Hoc.

${ }^{*} p<.05$.

muestras en función del sexo y la edad (ver Tabla 2). Los datos sugieren, por una parte, un alto nivel de relación directa de la resiliencia con el autoconcepto general y, por otra parte, una relación de menor magnitud con el apoyo social percibido. Por último, las correlaciones parciales controlando sexo y edad muestran resultados similares, lo que significa que ambas variables sociopersonales no poseen un efecto mediador.

\section{Adolescentes resilientes: niveles de autoconceptoy apoyo social percibido}

Con el objetivo de contrastar los resultados obtenidos en las correlaciones realizadas, se analizó si los adolescentes que presentaban puntuaciones altas en resiliencia mostraban diferencias significativas en autoconcepto y apoyo social al compararlos con aquellos que presentaron puntuaciones medias o bajas. Primero se agrupó la muestra en tres perfiles en función de las puntuaciones obtenidas en la escala CD-RISC: resiliencia baja (percentil $<20$ ), resiliencia media (percentil 20-80) y resiliencia alta (percentil > 80). Después, se realizaron análisis descriptivos (medias y desviaciones típicas), de varianza en función del nivel de resiliencia, del tamaño del efecto (Eta) y post-hoc de comparaciones múltiples (Bonferroni). Los resultados se presentan en la Tabla 3.

Tal y como se puede comprobar en la Tabla 3, aquellos adolescentes que manifestaron puntuaciones altas en resiliencia (percentil $>80$ ) comparados con los de resiliencia baja (percentil $<20$ ) disfrutaban de un auto- concepto más alto y percibían un mayor apoyo por parte de la familia, las amistades y el profesorado. El autoconcepto presenta una magnitud del tamaño del efecto elevada, el apoyo de la familia y el apoyo del profesorado ejercen un efecto moderado, y el apoyo de las amistades un efecto pequeño. Las pruebas post-hoc parecen sugerir que cualquier incremento en la resiliencia trae consigo invariablemente un alto nivel de autoconcepto y de apoyo social, es decir, siempre se dan diferencias significativas $(p<.05)$ entre todos los grupos de la resiliencia.

\section{Variables predictoras de la resiliencia}

Con el objetivo de explorar las variables que predicen la resiliencia en la adolescencia, se realizó un análisis de regresión lineal múltiple (método pasos sucesivos) que somete a prueba la predicción del autoconcepto y las facetas de apoyo social sobre la resiliencia, cuyos resultados se presentan en la Tabla 4.

Todas las variables predictoras propuestas son significativas $(p<.05)$ en la predicción de la resiliencia para la muestra en su conjunto. Los coeficientes Beta estandarizados señalan que el autoconcepto $(\beta=.387)$ es la variable con mayor capacidad explicativa. Alto nivel de autoconcepto, alto nivel de apoyo de la familia, alto nivel de apoyo del profesorado y alto nivel de apoyo de las amistades resultaron variables predictoras de la resiliencia, llegando a explicar en su conjunto el $23.3 \%$ $\left(\Delta R^{2}=.233\right)$ de la variable dependiente. En ambas muestras en función del sexo, las variables autoconcepto, 
Tabla 4

Capacidad Predictiva del Autoconcepto y el Apoyo Social sobre la Resiliencia

\begin{tabular}{|c|c|c|c|c|c|c|}
\hline Variable predictora & $R^{2}$ & $\Delta R^{2}$ & Error típico & Constante & $\beta$ & $t$ \\
\hline \multicolumn{7}{|c|}{$\begin{array}{c}\text { Muestra total } \\
\text { Resiliencia }\left(F_{(4,1249)}=94.81^{*}\right)\end{array}$} \\
\hline Autoconcepto & .206 & .206 & .074 & 49.70 & .387 & $14.36^{*}$ \\
\hline Apoyo familiar & .221 & .221 & .052 & 44.36 & .084 & $2.93^{*}$ \\
\hline Apoyo profesorado & .229 & .229 & .041 & 42.59 & .094 & $3.56^{*}$ \\
\hline Apoyo amistades & .233 & .233 & .055 & 40.20 & .073 & $2.72^{*}$ \\
\hline \multicolumn{7}{|c|}{$\begin{array}{c}\text { Chicos } \\
\text { Resiliencia }\left(F_{(3,611)}=55.36^{\star}\right)\end{array}$} \\
\hline Autoconcepto & .173 & .171 & .108 & 51.54 & .359 & $9.62^{*}$ \\
\hline Apoyo amistades & .207 & .204 & .074 & 44.01 & .183 & $4.93^{*}$ \\
\hline Apoyo profesorado & .215 & .211 & .053 & 41.57 & .090 & $2.48^{*}$ \\
\hline \multicolumn{7}{|c|}{$\begin{array}{c}\text { Chicas } \\
\text { encia }\left(F_{(4,637)}=55.25^{\star}\right)\end{array}$} \\
\hline Autoconcepto & .216 & .215 & .099 & 49.52 & .359 & $9.27^{*}$ \\
\hline Apoyo profesorado & .240 & .238 & .060 & 44.77 & .129 & $3.50^{*}$ \\
\hline Apoyo amistades & .252 & .248 & .082 & 39.53 & .096 & $2.59^{*}$ \\
\hline Apoyo familiar & .259 & .254 & .070 & 37.21 & .097 & $2.48^{*}$ \\
\hline \multicolumn{7}{|c|}{$\begin{array}{c}12-13 \text { años } \\
\text { Resiliencia }\left(F_{24,503)}=49.30^{*}\right)\end{array}$} \\
\hline Autoconcepto & .143 & .142 & .121 & 54.10 & .327 & $7.56^{*}$ \\
\hline Apoyo familiar & .164 & .161 & .079 & 47.62 & .154 & $3.54^{*}$ \\
\hline \multicolumn{7}{|c|}{$\begin{array}{c}14-15 \text { años } \\
\operatorname{ncia}\left(F_{(3,745)}=91.11^{*}\right)\end{array}$} \\
\hline Autoconcepto & .246 & .245 & .089 & 47.31 & .453 & $13.75^{*}$ \\
\hline Apoyo profesorado & .261 & .259 & .050 & 43.48 & .118 & $3.71^{*}$ \\
\hline Apoyo amistades & .269 & .266 & .069 & 39.37 & .094 & $2.88^{*}$ \\
\hline
\end{tabular}

${ }^{*} p<.05$.

apoyo del profesorado y apoyo de las amistades fueron significativas para la variable criterio resiliencia, mientras que el apoyo familiar sólo lo fue para la muestra de sexo femenino. Los coeficientes de regresión estandarizados Beta indican que el autoconcepto (chicos: $\beta=.359$; chicas: $\beta=.359$ ) es la variable con más peso en el modelo, seguida del apoyo de las amistades en el caso de los chicos $(\beta=.183)$ y del apoyo del profesorado $(\beta=$ .129) en el caso de las chicas. Resultaron predictoras de la resiliencia tres variables (alto nivel de autoconcepto, alto nivel de apoyo de las amistades y alto nivel de apoyo del profesorado) que explican el $21.1 \%$ $\left(\Delta R^{2}=.211\right)$ de la varianza para los chicos y el $25.4 \%$ para las chicas si se añade alto nivel de apoyo de la familia. En último lugar, en las muestras en función de la edad puede observarse que las variables autoconcepto y apoyo de la familia son relevantes a la hora de predecir la resiliencia en el grupo de edad de 12-13 años, mientras que en la muestra de adolescentes de 14-15 años se identifican como variables significativas para la variable criterio resiliencia el autoconcepto, el apoyo del profesorado y el apoyo de las amistades. Los coeficientes Beta estandarizados indican que es el autoconcepto la variable con mayor capacidad explicativa en ambas muestras (12-13 años: $\beta=.327$; $14-15$ años: $\beta=.453$ ). La conjunción de alto nivel de autoconcepto y alto nivel de apoyo de la familia llegan a explicar un $16.1 \%\left(\Delta R^{2}=\right.$ .161) de la variable independiente en los jóvenes de 1213 años, mientras que en el grupo de 14-15 años alto nivel de autoconcepto, alto nivel de apoyo del profesorado y alto nivel de apoyo de las amistades se identifican como variables predictoras de la resiliencia, explicando el $26.6 \%$ de la varianza $\left(\Delta R^{2}=.266\right)$. 


\section{Discusión}

La acertada crítica realizada por parte de la psicología positiva a la excesiva preocupación por los déficits o factores asociados a conductas problemáticas (Seligman, 2011) ha dado lugar a un enfoque predominante en la actualidad que, dejando atrás las limitaciones, traslada el énfasis a los elementos que facilitan el desarrollo óptimo de los adolescentes. De manera opuesta al modelo centrado en el riesgo, desde el modelo basado en las fortalezas se considera que todo adolescente posee el potencial para lograr un desarrollo exitoso y la capacidad para evolucionar de manera equilibrada (Sesma, Mannes y Scales, 2013). Por ello, en este estudio se argumenta a favor del lado saludable y ajustado del desarrollo adolescente incluyendo únicamente indicadores positivos de adaptación y planteando como objetivo el análisis de las relaciones de la resiliencia con el autoconcepto y el apoyo social percibido (apoyo de la familia, amistades y profesorado).

La asociación positiva entre la resiliencia y el autoconcepto-autoestima es una cuestión que ha sido consistentemente corroborada a nivel empírico (Benetti y Kambouropoulos, 2006; Dumont y Provost, 1999; Karatas y Savi Cakar, 2011; Kidd y Shahar, 2008). El presente trabajo no sólo ratifica el estrecho vínculo entre la autopercepción positiva y la resiliencia, sino que además confirma que el autoconcepto de los adolescentes predice de forma positiva la conducta resiliente. Estos resultados confirman los hallazgos obtenidos en estudios previos que también han señalado la visión positiva de uno mismo como factor predictor del comportamiento resiliente en niños y jóvenes (Cardozo y Alderete, 2009; Masten 2006; Wright et al., 2013).

A nivel general, se constata la relevancia de las fuentes de soporte social en la superación de la adversidad durante el periodo de la adolescencia (Wright et al., 2013). Los resultados obtenidos en la presente investigación sugieren una conexión directa de la resiliencia con el apoyo familiar, el apoyo de las amistades y el apoyo del profesorado, poniéndose de relieve que los estudiantes resilientes presentan puntuaciones significativamente más altas en apoyo social que los estudiantes no resilientes. Estos datos confirman el planteamiento de Masten (2006), quien identifica los lazos con los iguales y con determinadas figuras de adultos mentores (red fa- miliar y profesorado) como factores asociados al comportamiento resiliente durante la infancia y la adolescencia. Asimismo, los resultados se encuentran en la misma línea que las escasas aportaciones empíricas disponibles que señalan el significativo vínculo positivo entre apoyo social y resiliencia (Bozack, 2014; Everall et al., 2006; Tusaie, Puskar y Sereika, 2007). Por otro lado, en este estudio se identifican las tres facetas de apoyo social (apoyo familiar, apoyo de las amistades y apoyo del profesorado) como predictores de la resiliencia, lo que permite corroborar la investigación de Sun et al. (2013), cuyas conclusiones hacen referencia a que un alto nivel de apoyo social contribuye a incrementar la resiliencia.

En síntesis, el análisis de regresión múltiple sometido a verificación empírica en esta investigación ha permitido constatar el reconocimiento del autoconcepto y el apoyo social percibido de la familia, amistades y profesorado como elementos decisivos en la predicción de la resiliencia de los adolescentes de ambos sexos. La relación más destacada es la que mantiene el autoconcepto con la resiliencia, siendo su influencia especialmente relevante. Se comprueba también que el apoyo de la familia, el círculo de amistades y el profesorado determinan de manera diferenciada la resiliencia, constituyéndose el apoyo familiar percibido como la fuente de soporte social con más peso en el conjunto de la muestra. Además, este análisis destinado a detectar factores personales y contextuales que debutan de manera combinada en la predicción de la resiliencia, revela que es el autoconcepto la variable que mayor capacidad de predicción presenta; resultados que coinciden con el estudio de Cardozo y Alderete (2009), en el que de un conjunto de aspectos sociales e individuales el autoconcepto era la variable que mejor predice la resiliencia.

Por tanto, si para los escolares adolescentes son componentes determinantes de la conducta resiliente el autoconcepto y el soporte social, los resultados poseen implicaciones educativas a nivel práctico que permiten sugerir la importancia de implementar programas de intervención psicológica en el ámbito escolar con el objeto de promocionar la aceptación de uno mismo. También desde la escuela debe prestarse atención a la mejora de las relaciones sociales (familiares, de amistad y con la figura docente) para lograr un afrontamiento adecuado de 
la adversidad. Las intervenciones enfocadas a incrementar los niveles de la resiliencia a través del fomento de recursos personales ofrecen a los profesionales de la psicología una oportunidad de movimiento más allá del modelo del déficit en el ámbito aplicado (Tolan y Carrigan, 2011). En este sentido, desde el enfoque centrado en la promoción de las fortalezas humanas o atributos psicológicos positivos (orientado a poblaciones adolescentes clínicas y no clínicas) se apuesta por intervenciones preventivas que sirvan para equipar a los jóvenes con aquellas habilidades necesarias para la gestión adecuada de posibles problemas o dificultades (Brooks y Brooks, 2014). De manera específica, se ha demostrado la efectividad de programas de promoción de la resiliencia en el contexto escolar que impulsan el empoderamiento y las autopercepciones positivas considerando factores ambientales como las relaciones sociales (Song, Sikorski, Doll y Sikorski, 2014).

Finalmente, como limitación del estudio cabe destacar la naturaleza transversal del trabajo y el limitado significado de la capacidad predictiva hallada, que no permite afirmar que las relaciones halladas sean de tipo causal al hacer referencia a una incidencia estadística. Por tanto, futuras investigaciones podrían profundizar en la realización de un estudio longitudinal de carácter prospectivo, además del uso de análisis estadísticos como los modelos de ecuaciones estructurales. Además, se ha de tener en cuenta que los resultados de este trabajo son exploratorios y se ajustan a una muestra de estudiantes de secundaria con edades comprendidas entre los 12 y los 15 años de edad, por lo que no son generalizables a adolescentes de otras edades; en este sentido, se debería ampliar el estudio a otras edades.

\section{Referencias}

Becoña, E. (2006). Resiliencia: Definición, características y utilidad del concepto. Revista de Psicopatología y Psicología Clínica, 11(3), 125146.

Benetti, C. y Kambouropoulos, N. (2006). Affectregulated indirect effects of trait anxiety and trait resilience on self-esteem. Personality and Individual Differences, 41, 341-352. doi: http://dx.doi.org/10.1016/j.paid.2006.01.015

Bobes, J., Bascaran, M. T., García-Portilla, M. P., Bousoño, M., Sáiz, P. A. y Wallance, D. H. (2001). Banco de instrumentos básicos de psiquiatría clínica. Barcelona, España: Psquiatría ED.

Bozak, S. (2014). College students' sense of belonging and social support: Potential factors in resilience. Dissertation Abstracts International, 74.

Brooks, R. y Brooks, S. (2014). Creating resilient mindsets in children and adolescents: A strengthbased approach for clinical and nonclinical populations. En S. Prince-Embury, D. H. Saklofske, S. Prince-Embury y D. H. Saklofske (Eds.), Resilience interventions for youth in diverse populations (pp. 59-82). New York, NY, US: Springer Science + Business Media.

Campbell-Sills, L., Cohan, S. L. y Stein, M. B. (2006). Relationship of resilience to personality, coping, and psychiatric symptoms in young adults. Behaviour Research and Therapy, 44(4), 585-599. doi: http://dx.doi.org/10.1016/j.brat.2005.05.001

Cardozo, G. y Alderete, A. M. (2009). Adolescentes en riesgo psicosocial y resiliencia. Psicología desde el Caribe, 23, 148-182.

Chu, P. S., Saucier, D. A. y Hafner, E. (2010). Metaanalysis of the relationships between social 
support and well-being in children and adolescents. Journal of Social and Clinical Psychology, 29(6), 624-645. doi: http://dx.doi.org/10.1521/jscp.2010.29.6.624

Cohen, S. (2004). Social relationships and health. American Psychologist, 59(8), 676-684. doi: http://dx.doi.org/10.1037/0003-066X.59.8.676

Connor, K. M. y Davidson, J. T. (2003). Development of a new resilience scale: The Connor-Davidson Resilience Scale (CD-RISC). Depression and Anxiety, 18(2), 76-82. doi: http://dx.doi.org/10.1002/da.10113

Dumont, M. y Provost, M. A. (1999). Resilience in adolescents: Protective role of social support, coping strategies, self-esteem, and social activities on experience of stress and depression. Journal of Youth and Adolescence, 28(3), 343-363. doi: http://dx.doi.org/10.1023/A:1021637011732

Everall, R. D., Altrows, K. y Paulson, B. L. (2006). Creating a Future: A study of resilience in suicidal female adolescents. Journal of Counseling and Development, 84(4), 461-471. doi: http://dx.doi.org/10.1002/j.1556-

6678.2006.tb00430.x

Fergus, S. y Zimmerman, M. A. (2005). Adolescent resilience: A framework for understanding healthy development in the face of risk. Annual Review of Public Health, 26, 399-419. doi: http://dx.doi.org/10.1146/annurev.pubhealth.26.02 1304.144357

Fernández-Zabala, A., Goñi, E., Camino, I. y Zulaika, L. M. (en prensa). El contexto familiar y escolar en la implicación escolar. European Journal of Education and Psychology, 9(1).

Fernández-Zabala, A., Goñi, E., RodríguezFernandez, A. y Goñi A. (2015). Un nuevo cuestionario en castellano con escalas de las dimensiones del autoconcepto. Revista Mexicana de Psicología, 32(2), 149-159.

Fletcher, D. y Sarkar, M. (2013). Psychological resilience: A review and critique of definitions, concepts, and theory. European Psychologist, 18(1), 12-23. doi: http://dx.doi.org/10.1027/10169040/a000124

Fuentes, M. C., García, J. F., Gracia, E. y Lila, M. (2011). Autoconcepto y ajuste psicosocial en la adolescencia. Psicothema, 23(1), 7-12.

Garaigordobil, M., Pérez, J. y Mozaz, M. (2008). Selfconcept, self-esteem and psychopathological symptoms. Psicothema, 20(1), 114-123.

Garmezy, N., Masten, A. S. y Tellegen, A. (1984). The study of stress and competence in children: A building block for developmental psychopathology. Child Development, 55(1), 97111. doi: http://dx.doi.org/10.2307/1129837

Giménez, M., Valverde, C. V. y Hervás, G. (2010). El análisis de las fortalezas psicológicas en la adolescencia: Más allá de los modelos de vulnerabilidad. Psychology, Society and Education, 2(2), 97-116.

González, M. y Landero, R. (2014). Propiedades psicométricas de la escala de Apoyo Social Familiar y de Amigos (AFA-R) en una muestra de estudiantes. Acta de Investigación Psicológica, 4(2), 1469-1480. doi: http://dx.doi.org/10.1016/S2007-4719(14)70387-4

Inglés, C., Martínez-Monteagudo, M., GarcíaFernández, J., Valle, A. y Castejón, J. (2014). Perfiles de orientaciones de metas y autoconcepto de estudiantes de Educación Secundaria. Revista de Psicodidáctica, 20(1), 99-116. doi: http://dx.doi.org/10.1387/RevPsicodidact.1023

Jorgensen, I. E. y Seedat, S. (2008). Factor structure of the Connor-Davidson resilience scale in South 
African adolescents. International Journal of Adolescent Medicine and Health, 20(1), 23-32. doi: http://dx.doi.org/10.1515/ijamh.2008.20.1.2

Karatas, F. y Savi Cakar, F. (2011). Self-esteem and hopelessness, and resiliency: an exploratory study of adolescents in Turkey. International Education Studies, 4(4), 84-91. doi: http://dx.doi.org/10.5539/ies.v4n4p84.

Kidd, S. y Shahar, G. (2008). Resilience in homeless youth: The key role of self-esteem. American Journal of Orthopsychiatry, 78(2), 163-172. doi: http://dx.doi.org/10.1037/0002-9432.78.2.163.

Landero, R. y González, M. (2008). Escala de Apoyo Social Percibido de Familia y Amigos (AFA). Documento interno de la Facultad de Psicología, Universidad Autónoma de Nuevo León.

Lerner, R. M., Bowers, E. P., Geldhof, G. J., Gestsdóttir, S. y DeSouza, L. (2012). Promoting positive youth development in the face of contextual changes and challenges: The roles of individual strengths and ecological assets. New Directions for Youth Development, 2012(135), 119-128. doi: http://dx.doi.org/10.1002/yd.20034

Lerner, J. V., Phelps, E., Forman, Y. y Bowers, E. P. (2009). Positive youth development. En R. M. Lerner, L. Steinberg, R. M. Lerner y L. Steinberg (Eds.), Handbook of adolescent psychology, Vol 1: Individual bases of adolescent development (pp. 524-558). Hoboken, NJ, US.: John Wiley.

Liu, Y., Wang, Z. y Lü, W. (2013). Resilience and affect balance as mediators between trait emotional intelligence and life satisfaction. Personality and Individual Differences, 54(7), 850-855. doi: http://dx.doi.org/10.1016/j.paid.2012.12.010

Luthar, S. S., Cicchetti, D. y Becker, B. (2000). The construct of resilience: A critical evaluation and guidelines for future work. Child Development,
71(3), 543-562. doi: http://dx.doi.org/10.1111/1467-8624.00164

Luthar, S. S., Lyman, E. L. y Crossman, E. J. (2014). Resilience and positive psychology. En M. Lewis y Rudolph (Eds.), Handbook of Developmental Psychopathology (pp. 125-140). USA: Springer. doi: http://dx.doi.org/10.1007/978-1-4614-9608$3+7$

Masten (2006). Promoting resilience in development: A general framework for systems of care. En R. J. Flynn, P. M, Dudding y J. G. Barber (Eds.), Promoting resilience in child welfare (pp. 3-17). Ottawa, ON, Canada: University of Ottawa Press.

Masten, A. S. (2014). Invited commentary: Resilience and positive youth development frameworks in developmental science. Journal of Youth and Adolescence, 43(6), 1018-1024. doi: http://dx.doi.org/10.1007/s10964-014-0118-7

Masten, A. S. y Tellegen, A. (2012). Resilience in developmental psychopathology: Contributions of the Project Competence Longitudinal Study. Development and Psychopathology, 24(2), 345-361. doi: http://dx.doi.org/10.1017/S095457941200003X

Moore, K. y Lippman, L. H. (2005). What do children need to flourish: Conceptualizing and measuring indicators of positive development. New York, NY, US: Springer Science + Business Media. doi: http://dx.doi.org/10.1007/b100487

Musitu, G. y Cava, M. J. (2003). El rol del apoyo social en el ajuste de los adolescentes. Intervención Psicosocial, 12(2), 179-192.

Moreno, C., Muñoz-Tinoco, V., Pérez, P., SánchezQueija, I., Granado M. C., Ramos, P. y Rivera, F. (2008). Desarrollo adolescente y salud. Resultados del Estudio HBSC-2006 con chicos y chicas españoles de 11 a 17 años. Madrid, España: Ministerio de Sanidad y Consumo. 
Oliva, A., Jiménez, J. M., Parra, Á. y SánchezQueija, I. (2008). Acontecimientos vitales estresantes, resiliencia y ajuste adolescente. Revista de Psicopatología y Psicología Clínica, 13(1), 53-62.

Oliva, A., Ríos, M., Antolín, L., Parra, Á., Hernando, Á. y Pertegal, M. (2010). Beyond the deficit: Building a model of positive youth development. Infancia y Aprendizaje, 33(2), 223-234. doi: http://dx.doi.org/10.1174/021037010791114562

Olsson, C. A., Bond, L., Burns, J. M., VellaBrodrick, D. A. y Sawyer, S. M. (2003). Adolescent resilience: A concept analysis. Journal of Adolescence, 26(1), 1-11. doi: http://dx.doi.org/:10.1016/S0140-1971(02)001185

Rodríguez-Fernández, A., Droguett, L. y Revuelta, L. (2012). Ajuste escolar y personal en la adolescencia: El papel del autoconcepto académico y del apoyo social percibido. Revista de Psicodidáctica, 17(2), 397-413. doi: http://dx.doi.org/ 0.1387/Rev.Psicodidact.3002

Rodríguez-Fernández, A., Ramos-Díaz, E., Madariaga, J. M., Arribillaga, A. y Galende, N. (en prensa). Steps in the construction and verification of an explanatory model of psychosocial adjustment. European Journal of Education and Psychology, 9(1).

Rohner, R. P. y Carrasco, M. Á. (2014). Teoría de la Aceptación-Rechazo Interpersonal (IPARTheory): Bases Conceptuales, Método y Evidencia Empírica. Acción Psicológica,11(2). doi: http://dx.doi.org/10.5944/ap.11.2.14172

Rutter, M. (2007). Resilience, competence, and coping. Child Abuse and Neglect, 31(3), 205-209. doi:http://dx.doi.org/10.1016/j.chiabu.2007.02.00 1
Seligman, M. E. (2011). Flourish: A new understanding of happiness and well-being and how to achieve them. Londres, UK: Nicholas Brealey Publishing.

Seligman, M. P. y Csikszentmihalyi, M. (2000). Positive psychology: An introduction. American Psychologist, 55(1), 5-14. doi: http://dx.doi.org/10.1037/0003-066X.55.1.5

Sesma, A. J., Mannes, M. y Scales, P. C. (2013). Positive adaptation, resilience and the developmental assets framework. En S. Goldstein, R. B. Brooks, S. Goldstein y R. B. Brooks (Eds.), Handbook of resilience in children (pp. 427-442). New York, NY, US: Springer Science + Business Media. doi: http://dx.doi.org/10.1007/978-1-4614-3661-4_25

Shavelson, J., Hubner, J. J. y Stanton, G. J. (1976). Self concept: validation of construct interpretations. Review of Educational Research, 46(3), 407-441. doi: http://dx.doi.org/10.2307/1170010

Sheldon, K. M. y King, L. (2001). Why positive psychology is necessary. American Psychologist, 56, 216-217. doi: http://dx.doi.org/10.1037/0003066X.56.3.216

Song, S. Y., Sikorski, J., Doll, B. y Sikorski, M. (2014). Enhancing classroom resilience with ClassMaps Consultation. En S. Prince-Embury, D. H. Saklofske, S. Prince-Embury y D. H. Saklofske (Eds.), Resilience interventions for youth in diverse populations (pp. 203-215). New York, NY, US: $\quad$ Springer Science. doi: http://dx.doi.org/10.1007/978-1-4939-0542-3_10

Stumblingbear-Riddle, G. y Romans, J. C. (2012). Resilience among urban America Indian adolescents: Exploration into the role of culture, self-esteem, subjective well-being, and social support. American Indian and Alaska Native 
Mental Health Research, 19(2), 1-19. doi: http://dx.doi.org/10.5820/aian.1902.2012.1

Sun, S., Guan, Y., Qin, Y., Zhang, L. y Fan, F. (2013). Social support and emotional-behavioral problems: Resilience as a mediator and moderator. Chinese Journal of Clinical Psychology, 21(1), 114-118.

Tian, L., Liu, B., Huang, S. y Huebner, E. S. (2013). Perceived social support and school well-being among Chinese early and middle adolescents: The mediational role of self-esteem. Social Indicators Research, 113(3), 991-1008.

Toland, J. y Carrigan, D. (2011). Educational psychology and resilience: New concept, new opportunities. School Psychology International, 32(1), 95-106. doi: http://dx.doi.org/10.1177/0143034310397284

Tusaie, K., Puskar, K. y Sereika, S. M. (2007). A predictive and moderating model of psychosocial resilience in adolescents. Journal of Nursing Scholarship, 39(1), 54-60. doi: http://dx.doi.org/10.1111/j.15475069.2007.00143.x

Werner, E. E. y Smith, R. S. (1992). Overcoming the odds: High risk children from birth to adulthood. Ithaca, NY: Cornell University Press.

Wright, M., Masten, A. S. y Narayan, A. J. (2013). Resilience processes in development: Four waves of research on positive adaptation in the context of adversity. En S. Goldstein y R. B. Brooks (Eds.), Handbook of resilience in children (pp. 1537). New York, NY US: Springer Science + Business Media. doi: http://dx.doi.org/10.1007/978-1-4614-3661-4

Yu, X. N., Lau, J. T., Mak, W. W., Zhang, J. y Lui, W. W. (2011). Factor structure and psychometric properties of the Connor-Davidson Resilience Scale among Chinese adolescents. Comprehensive
Psychiatry, 52(2), 218-224. doi: http://dx.doi.org/10.1016/j.comppsych.2010.05.01 0 